\title{
General Time-Division AltBOC Modulation Technique for GNSS Signals
}

\author{
Zhihui ZHOU, Zuping TANG, Jiaolong WEI, Xuan XIA
}

School of Electronic Information and Communications, Huazhong University of Science and Technology, Luoyu Road 1037, 430074 Wuhan, China

zhih_zhou@163.com, tang_zuping@hust.edu.cn,jlwei@mail.hust.edu.cn,26329550@qq.com

Submitted June 6, 2017 / Accepted November 24, 2017

\begin{abstract}
In this paper, a general time-division alternate binary offset carrier (GTD-AltBOC) modulation method is proposed, which is an extension of TD-AltBOC and time-multiplexed offset-carrier quadrature phase shift keying (TMOC-QPSK) with high design flexibility. In this method, binary complex subcarriers and a time-division technique with flexible time slot assignment are used to achieve constant envelope modulation of the signal components with a variable PAR. The underlying principle of GTD-AltBOC and the constraints related to the PAR are investigated. For the generation of GTD-AltBOC signals, a lookup table (LUT)-based scheme is presented; the minimum required clock rate is half or less of that for existing non-time-division methods. The receiver processing complexities are analyzed for three typical receiving modes, and the power spectral densities (PSDs), cross-correlation functions, multiplexing efficiencies and code-tracking performance are simulated; the results show that GTD-AltBOC enables a significant decrease in receiving complexity compared with existing methods while maintaining high performance in terms of multiplexing efficiency and code tracking.
\end{abstract}

\section{Keywords}

Global Navigation Satellite System (GNSS), AltBOC, modulation, time division, signal design

\section{Introduction}

With the continuing development of global navigation satellite systems (GNSSs), the frequency allocation schemes for GNSS services are becoming crowded, and newly emerging systems, such as Galileo and Beidou, are forced to transmit their navigation service signals in disperse frequency bands. If the interval between two bands is very small, such as that between the Galileo E5a and E5b bands [1] or that between the Beidou B2a and B2b bands [2], the need for signal transmission in those bands using a unique high-power amplifier (HPA) is urgent [3]. Research on combining several direct sequence spread spectrum (DSSS) signals at two adjacent frequencies into a constant envelope composite sig- nal has become critically important, and many methods have been proposed to solve this problem.

Alternate binary offset carrier (AltBOC) [1], [3] modulation is a widely known dual-frequency constant envelope modulation technique that is successfully used in the E5 band of the Galileo system. AltBOC signals can achieve unprecedented tracking and anti-multipath performance. However, the powers of the four signal components must be equal for AltBOC modulation, which imposes a strict constraint on signal design. Moreover, the processing complexity for full-band AltBOC signals is somewhat high for GNSS hardware receivers, especially portable receivers. To reduce this complexity, time-division AltBOC (TD-AltBOC) [4] and time-multiplexed offset-carrier quadrature phase shift keying (TMOC-QPSK) [5] have been proposed. In these two methods, a time-division technique is introduced to reduce the complexity. However, similar to AltBOC, these two methods also require the four signal components to have the same power.

For GNSS signal design, the power allocation between the data and pilot channels is an important factor that affects the overall system performance [6]. In most existing GNSS signals, such as GPS L2C and L5C [7], [8] and Galileo E1, E5a, and E5b [1], power is equally allocated between the data and pilot components. By contrast, for the GPS L1C signal, more power is allocated to the pilot component to enhance the signal tracking performance [9]. Therefore, for use in GNSS signal design, a modulation technique must have sufficient flexibility to allow the power allocation among the signal components to be adjusted to meet different demands. Generalized AltBOC [10] is an extension of AltBOC that is suitable for applications with different powers in the upper and lower sidebands while retaining a complexity similar to that of AltBOC, and its multiplexing efficiency is higher than that of AltBOC. In addition, Yao Z. and Lu M. proposed an asymmetric constant envelope BOC (ACE-BOC) technique [11], [12] for combining four signal components with arbitrary power allocation. However, for certain special power ratios, the complexity of ACE-BOC is significantly increased compared with that of AltBOC. To reduce the complexity of ACE-BOC, ACE-BOC with equal-length subcarrier seg- 
ments (ES ACE-BOC) [12] and ACE-BOC with bipolar subcarriers (BS-ACEBOC) [13] have been proposed. ES ACEBOC has a complexity similar to that of AltBOC, but it suffers from some loss in multiplexing efficiency. BS-ACEBOC enables a significant reduction in complexity compared with AltBOC, but at the cost of a severe decrease in multiplexing efficiency. As another alternative, Yan et al. proposed a general AltBOC (GAltBOC) [14] modulation scheme with an adjustable power allocation ratio (PAR). GAltBOC offers high flexibility in power allocation with a complexity identical to that of AltBOC, but its multiplexing efficiency is severely attenuated for some special PARs.

Overall, research related to dual-frequency constant envelope modulation tends to focus on three aspects: I. increasing the flexibility of power allocation, II. decreasing the complexity of signal generation and processing, and III. improving the multiplexing efficiency. In this paper, we extend TD-AltBOC and TMOC-QPSK to allow for a variable PAR by proposing a general time-division AltBOC modulation (GTD-AltBOC) technique that preserves the advantages of both TD-AltBOC and TMOC-QPSK in terms of complexity and performance. In this method, different PARs can be achieved by adjusting the time slots used for the transmission of each signal component. For the generation of GTD-AltBOC signals, we present a lookup table (LUT)based signal generation architecture. The complexity of the proposed GTD-AltBOC modulation technique is analyzed and compared with the complexities of existing methods. We also simulate the power spectral density (PSD) and cross-correlation function to verify the correctness of GTDAltBOC. The multiplexing efficiency and code-tracking performance are also analyzed and compared with those of existing methods.

The remainder of this paper is organized as follows. In Sec. 2, the principle of GTD-AltBOC modulation is investigated. In Sec. 3, we propose a LUT-based signal generation scheme and analyze the minimum required clock rate. In Sec. 4 , we discuss three typical receiving modes and analyze the complexity of each. Simulations and a performance analysis are reported in Sec. 5. Finally, conclusions are presented in the last section.

\section{Principle of GTD-AltBOC Modula- tion}

\subsection{Signal Model}

Similar to the Galileo E5 signals, we consider the following situation: There are four DSSS signal components, $s_{\mathrm{LD}}(t), s_{\mathrm{LP}}(t), s_{\mathrm{UD}}(t)$, and $s_{\mathrm{UP}}(t)$, to be transmitted at a carrier frequency $f_{0}$. Here, $s_{\mathrm{LD}}(t)$ and $s_{\mathrm{LP}}(t)$ are the data component and pilot component, respectively, in the lower sideband, the center frequency of which is $f_{0}-f_{\mathrm{sc}}$. We use $f_{\mathrm{sc}}$ to denote the subcarrier frequency. Similarly, $s_{\mathrm{UD}}(t)$ and $s_{\mathrm{UP}}(t)$ are the data component and pilot component in the upper sideband, the center frequency of which is $f_{0}+f_{\mathrm{sc}}$. The data component is the signal component that is modulated by the navigation data, and the pilot component is the signal component without navigation data. To achieve dual-frequency signal modulation and ensure that the two sidebands can carry different information, we use binary complex subcarriers to shift the signal components to their corresponding frequencies. The binary complex subcarriers are expressed as

$$
\begin{aligned}
S C_{\mathrm{L}}(t) & =S C_{\cos }(t)-\mathrm{j} S C_{\mathrm{sin}}(t), \\
& =\operatorname{sign}\left(\cos \left(2 \pi f_{\mathrm{sc}} t\right)\right)-\mathrm{j} \operatorname{sign}\left(\sin \left(2 \pi f_{\mathrm{sc}} t\right)\right), \\
S C_{\mathrm{U}}(t) & =S C_{\cos }(t)+\mathrm{j} S C_{\mathrm{sin}}(t), \\
& =\operatorname{sign}\left(\cos \left(2 \pi f_{\mathrm{sc}} t\right)\right)+\mathrm{j} \operatorname{sign}\left(\sin \left(2 \pi f_{\mathrm{sc}} t\right)\right)
\end{aligned}
$$

where $\operatorname{sign}()$ is the sign function and $\mathrm{j}$ is the imaginary unit. Note that the real and imaginary parts of (1) are only two-level waveforms, whereas the subcarriers in AltBOC modulation have four levels [1].

For the proposed GTD-AltBOC modulation technique, the four signal components are transmitted alternately in two time slots, as is done in TD-AltBOC and TMOC-QPSK. This implies that a constant envelope 2-code AltBOC signal or a single-sideband offset-carrier signal is transmitted in each slot. Considering that two of the signal components are transmitted in the odd time slots and the two remaining components are transmitted in the even time slots, there are six possible time slot allocation schemes in total for the four signal components, as summarized in Tab. 1. However, as shown in Tab. 1, the only difference between the first three schemes and the last three schemes is that the signals transmitted in the even and odd time slots are exchanged. Therefore, there are only three independent time slot allocation schemes. Without loss of generality, we can assume that $s_{\mathrm{LD}}(t)$ is transmitted in the even time slots. Then, the other signal to be transmitted in the even time slots is chosen from among $s_{\mathrm{LP}}(t), s_{\mathrm{UD}}(t)$, and $s_{\mathrm{UP}}(t)$; these choices correspond to time slot allocation schemes 1,2 , and 3 , respectively, in Tab. 1. Figure 1 shows the schematic diagrams of the three time slot allocation schemes, where $P_{\mathrm{LD}}, P_{\mathrm{LP}}, P_{\mathrm{UD}}$ and $P_{\mathrm{UP}}$ denote the powers of $s_{\mathrm{LD}}(t), s_{\mathrm{LP}}(t), s_{\mathrm{UD}}(t)$, and $s_{\mathrm{UP}}(t)$, respectively. Note that a QPSK-like signal structure is preferred in each sideband to allow a receiver to process $s_{\mathrm{L}}(t)$ and GPS L5 simultaneously using a similar processing method.

- The two signal components in the lower sideband are transmitted in the even time slots, and the two signal components in the upper sideband are transmitted in the odd time slots. The corresponding schematic diagram is shown in Fig. 1 (a). This time slot allocation scheme is referred to as "GTD-AltBOC-LU". When the powers of all four signal components are equal, GTDAltBOC-LU is equivalent to TMOC-QPSK-ab [5].

- The data components in both sidebands are transmitted in the even time slots, and the pilot components in both sidebands are transmitted in the odd time slots. The corresponding schematic diagram is shown in Fig. 1 (b). This time slot allocation scheme is referred to as "GTDAltBOC-DP". When the powers of all four signal components are equal, the considered GTD-AltBOC-DP 


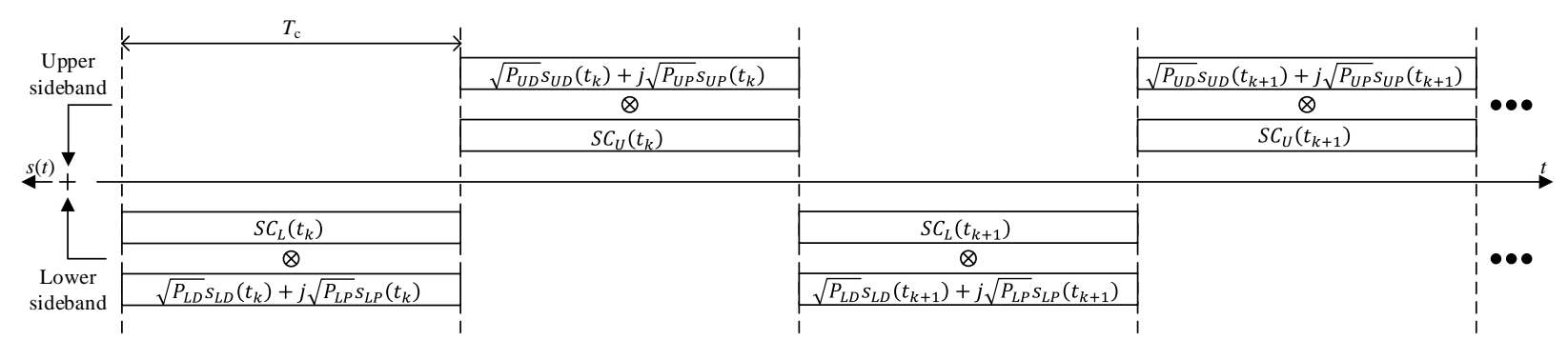

(a) GTD-AltBOC-LU

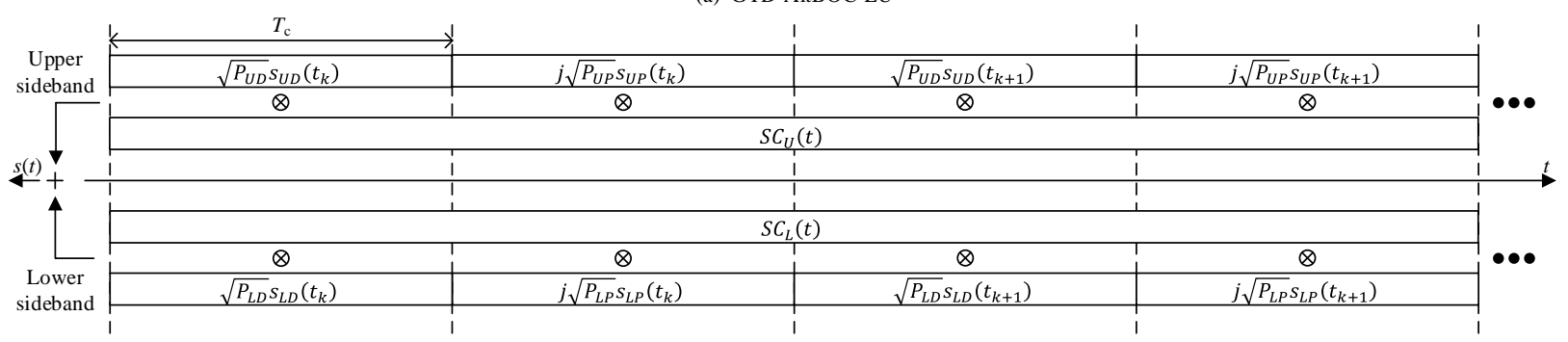

(b) GTD-AltBOC-DP

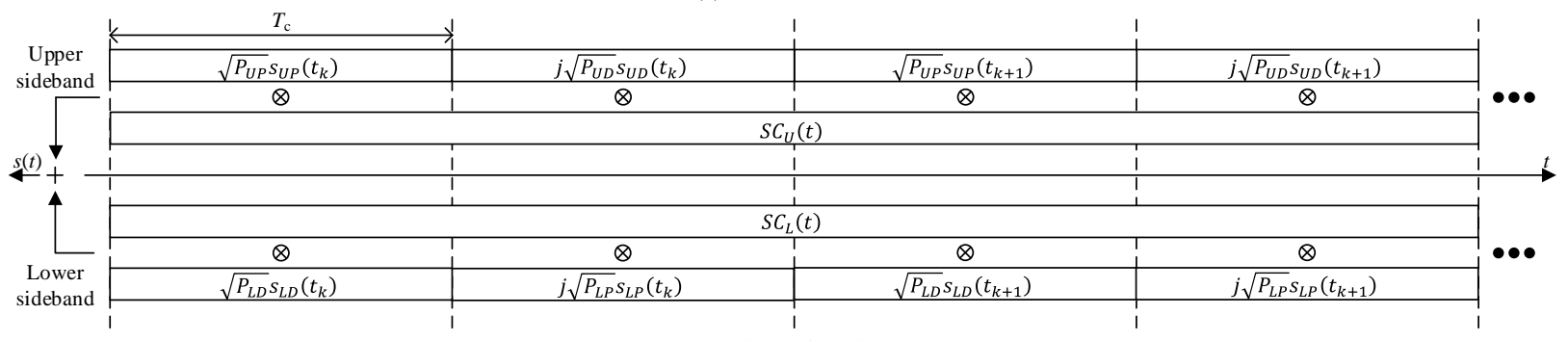

(c) GTD-AltBOC-X

Fig. 1. Schematic diagrams of the three time slot allocation schemes for GTD-AltBOC.

scheme is similar to TD-AltBOC [4]; the only difference is that the data components and the pilot components are orthogonal for GTD-AltBOC-DP, whereas they are in phase for TD-AltBOC. Note that the orthogonality between the data components and the pilot components is not a strong constraint; the in-phase case can also be an alternative scheme for GTD-AltBOC-DP. However, in this paper, which considers interoperability between GTD-AltBOC and GPS L5 signals, the orthogonal case is preferred.

- The data component in the lower sideband and the pilot component in the upper sideband are transmitted in the even time slots, and the pilot component in the lower sideband and the data component in the upper sideband are transmitted in the odd time slots. The corresponding schematic diagram is shown in Fig. 1 (c). This time slot allocation scheme is referred to as "GTD-AltBOC$\mathrm{X}$ ". When the powers of all four signal components are equal, GTD-AltBOC-X is equivalent to TMOC-QPSKIQ [5].

For clarity, we use the notation GTD-AltBOC $(m, n, r)$ for the proposed technique, where $\mathrm{r}$ is the PAR (in the form $\left.P_{\mathrm{LD}}: P_{\mathrm{LP}}: P_{\mathrm{UD}}: P_{\mathrm{UP}}\right)$ and $m$ and $n$ have the same meanings as in $\operatorname{AltBOC}(m, n)$, with the subcarrier frequency being $f_{\mathrm{sc}}=m \times 1.023 \mathrm{MHz}$ and the code rate being $R_{\mathrm{c}}=n \times 1.023$ $\mathrm{MHz}$. For example, GTD-AltBOC $(15,10,1: 3: 1: 3)$ indicates that the subcarrier frequency is $f_{\mathrm{sc}}=15 \times 1.023 \mathrm{MHz}$, the code rate is $R_{\mathrm{c}}=10 \times 1.023 \mathrm{MHz}$, and the PAR is $P_{\mathrm{LD}}: P_{\mathrm{LP}}: P_{\mathrm{UD}}: P_{\mathrm{UP}}=1: 3: 1: 3$.

\begin{tabular}{ccc}
\hline $\begin{array}{c}\text { Time slot } \\
\text { allocation scheme }\end{array}$ & Even time slots & Odd time slots \\
\hline 1 & $s_{\mathrm{LD}}(t) \& s_{\mathrm{LP}}(t)$ & $s_{\mathrm{UD}}(t) \& s_{\mathrm{UP}}(t)$ \\
2 & $s_{\mathrm{LD}}(t) \& s_{\mathrm{UD}}(t)$ & $s_{\mathrm{LP}}(t) \& s_{\mathrm{UP}}(t)$ \\
3 & $s_{\mathrm{LD}}(t) \& s_{\mathrm{UP}}(t)$ & $s_{\mathrm{LP}}(t) \& s_{\mathrm{UD}}(t)$ \\
4 & $s_{\mathrm{UD}}(t) \& s_{\mathrm{UP}}(t)$ & $s_{\mathrm{LD}}(t) \& s_{\mathrm{LP}}(t)$ \\
5 & $s_{\mathrm{LP}}(t) \& s_{\mathrm{UP}}(t)$ & $s_{\mathrm{LD}}(t) \& s_{\mathrm{UD}}(t)$ \\
6 & $s_{\mathrm{LP}}(t) \& s_{\mathrm{UD}}(t)$ & $s_{\mathrm{LD}}(t) \& s_{\mathrm{UP}}(t)$ \\
\hline
\end{tabular}

Tab. 1. The possible time slot allocation schemes for four signal components.

\subsection{Constraints on the PAR}

For the GTD-AltBOC-X scheme, as shown in Fig. 1 (c), the corresponding complex baseband signal is expressed as

$$
\begin{aligned}
s(t) & =\left[\sqrt{P_{\mathrm{LD}}} s_{\mathrm{LD}}(t)+\mathrm{j} \sqrt{P_{\mathrm{LP}}} s_{\mathrm{LP}}(t)\right] S C_{\mathrm{L}}(t) \\
& +\left[\mathrm{j} \sqrt{P_{\mathrm{UD}}} s_{\mathrm{UD}}(t)+\sqrt{P_{\mathrm{UP}}} s_{\mathrm{UP}}(t)\right] S C_{\mathrm{U}}(t),
\end{aligned}
$$




$$
\begin{aligned}
& s_{\mathrm{LD}}(t)=\sum_{l=-\infty}^{\infty} d_{\mathrm{LD}}(t) \sum_{k=1}^{L} C_{\mathrm{LD}}(k) p\left(t-(2 L l+2 k-2) T_{c}\right) \\
& s_{\mathrm{LP}}(t)=\sum_{l=-\infty}^{\infty} \sum_{k=1}^{L} C_{\mathrm{LP}}(k) p\left(t-(2 L l+2 k-1) T_{c}\right) \\
& s_{\mathrm{UD}}(t)=\sum_{l=-\infty}^{\infty} d_{\mathrm{UD}}(t) \sum_{k=1}^{L} C_{\mathrm{UD}}(k) p\left(t-(2 L l+2 k-1) T_{c}\right) \\
& s_{\mathrm{UP}}(t)=\sum_{l=-\infty}^{\infty} \sum_{k=1}^{L} C_{\mathrm{UP}}(k) p\left(t-(2 L l+2 k-2) T_{c}\right)
\end{aligned}
$$

where $d_{\mathrm{LD}}(t)$ and $d_{\mathrm{UD}}(t)$ denote the navigation data in the lower and upper sidebands, respectively; $C_{*}$ represents the pseudorandom noise (PRN) code of the corresponding signal component; $T_{\mathrm{c}}$ is the chip duration; $L$ denotes the length of the PRN codes; and $p(t)$ is the chip waveform, which is generally a rectangular function in a GNSS signal.

The envelope in even time slots is

$$
\begin{aligned}
A_{\mathrm{even}} & =\left|\sqrt{P_{\mathrm{LD}}} S_{\mathrm{LD}}(t) S C_{\mathrm{L}}(t)+\sqrt{P_{\mathrm{UP}}} S_{\mathrm{UP}}(t) S C_{\mathrm{U}}(t)\right|, \\
& =\sqrt{2\left(P_{\mathrm{LD}}+P_{\mathrm{UP}}\right)} .
\end{aligned}
$$

Similarly, the envelope in odd time slots is

$$
A_{\mathrm{odd}}=\sqrt{2\left(P_{\mathrm{LP}}+P_{\mathrm{UD}}\right)} .
$$

To satisfy the constant envelope condition, the envelopes in the even and odd time slots must be equal. Thus, the following relationship must hold for GTD-AltBOC-X:

$$
P_{\mathrm{LD}}+P_{\mathrm{UP}}=P_{\mathrm{LP}}+P_{\mathrm{UD}}
$$

By conducting analyses similar to that for GTDAltBOC-X, we can obtain the following constant envelope conditions for GTD-AltBOC-LU and GTD-AltBOC-DP, respectively:

$$
\begin{aligned}
& P_{\mathrm{LD}}+P_{\mathrm{LP}}=P_{\mathrm{UD}}+P_{\mathrm{UP}}, \\
& P_{\mathrm{LD}}+P_{\mathrm{UD}}=P_{\mathrm{LP}}+P_{\mathrm{UP}} .
\end{aligned}
$$

Consequently, when the PAR satisfies (6), (7) or (8), constant envelope modulation can be achieved using the proposed GTD-AltBOC technique. For example, consider two PARs of $1: 3: 1: 3$ and 1:1:3:3; these PARs have also been considered in the context of ACE-BOC [11], [12]. The PAR of 1:3:1:3 satisfies (6) and (7); therefore, a constantenvelope-modulated composite signal can be obtained using either GTD-AltBOC-LU or GTD-AltBOC-X. The PAR of 1:1:3:3 satisfies (6) and (8); therefore, a constant-envelopemodulated composite signal can be obtained using either GTD-AltBOC-DP or GTD-AltBOC-X. Figure 2 shows the constellation diagrams for GTD-AltBOC (15,10,1:3:1:3) and GTD-AltBOC(15,10,1:1:3:3), which are both 8-PSK-like signals.

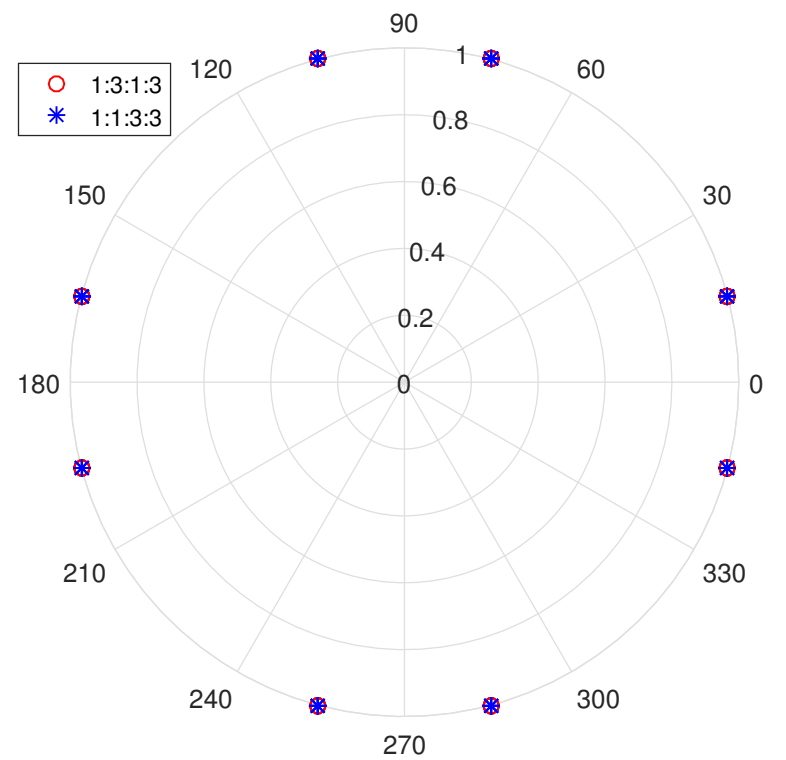

Fig. 2. The GTD-AltBOC constellation diagrams for PARs of $1: 3: 1: 3$ and $1: 1: 3: 3$.

\section{Signal Generation Scheme}

To generate a GTD-AltBOC signal, one approach is to generate the signal in accordance with its mathematical expression. To illustrate the process in detail, we use GTDAltBOC-X(m,n,r) as an example. By substituting (1) into (2), an in-phase component and a quadrature component can be obtained as follows:

$$
\begin{aligned}
s(t)= & \left(\sqrt{P_{\mathrm{LD}}} s_{\mathrm{LD}}(t) S C_{\mathrm{cos}}(t)+\sqrt{P_{\mathrm{LP}}} s_{\mathrm{LP}}(t) S C_{\mathrm{sin}}(t)\right. \\
& \left.+\sqrt{P_{\mathrm{UP}}} s_{\mathrm{UP}}(t) S C_{\mathrm{cos}}(t)-\sqrt{P_{\mathrm{UD}}} s_{\mathrm{UD}}(t) S C_{\mathrm{sin}}(t)\right) \\
& \mathrm{j}\left(\sqrt{P_{\mathrm{LP}}} S_{\mathrm{LP}}(t) S C_{\mathrm{cos}}(t)-\sqrt{P_{\mathrm{LD}}} s_{\mathrm{LD}}(t) S C_{\mathrm{sin}}(t)\right. \\
& \left.+\sqrt{P_{\mathrm{UD}}} S_{\mathrm{UD}}(t) S C_{\mathrm{cos}}(t)+\sqrt{P_{\mathrm{UP}}} S_{\mathrm{UP}}(t) S C_{\mathrm{sin}}(t)\right) .
\end{aligned}
$$

A GTD-AltBOC-X signal can be directly generated using several multipliers and adders configured in accordance with (9). This direct implementation is simple in principle and easy to implement in hardware. However, one disadvantage of direct generation is its lack of flexibility. As is well known, AltBOC signals are generated using a LUT-based method. Such a LUT-based method is a more flexible implementation structure for signal generation. Therefore, we also present a LUT-based method for GTD-AltBOC signal generation in this paper. Based on the mathematical expressions for the three time slot allocation schemes, we obtain the LUTs presented in Tab. 2 and Tab. 3. Note that the values in the LUTs for GTD-AltBOC-DP and GTD-AltBOC-X have similar expressions; hence, they are both represented in Tab. 3, where $x=$ 'D' and $y=$ 'P' for GTD-AltBOC-DP and $\mathrm{x}={ }^{\prime} \mathrm{P}$ ' and $\mathrm{y}={ }^{\prime} \mathrm{D}$ ' for GTD-AltBOC-X.

As seen from Tab. 2 and Tab. 3, the three LUTs are all two-dimensional tables of the same size and structure. Therefore, their hardware architectures will also be similar. Again, we consider GTD-AltBOC-X(m,n,r) as an example. Figure 3 shows the block diagram for GTD-AltBOC-X(m,n,r) 


\begin{tabular}{|c|c|c|c|c|c|c|c|c|c|}
\hline \multirow{2}{*}{\multicolumn{2}{|c|}{$\frac{\text { Even/odd selector }}{s_{\mathrm{LD}}}$}} & \multicolumn{4}{|c|}{ Even chip } & \multicolumn{4}{|c|}{ Odd chip } \\
\hline & & 1 & 1 & -1 & -1 & - & - & - & - \\
\hline & $s_{\mathrm{LP}}$ & 1 & -1 & 1 & -1 & - & - & - & - \\
\hline & $s_{\mathrm{UD}}$ & - & - & - & - & 1 & 1 & -1 & -1 \\
\hline & $s_{\mathrm{UP}}$ & - & - & - & - & 1 & -1 & 1 & -1 \\
\hline \multicolumn{2}{|c|}{$t \bmod T_{\mathrm{sc}}$} & \multicolumn{8}{|c|}{$\theta$ such that $s(t)=\exp (\mathrm{j} \theta)$, where $\theta_{\mathrm{e}}=\arctan \sqrt{\frac{P_{\mathrm{LP}}}{P_{\mathrm{LD}}}}$ and $\theta_{\mathrm{O}}=\arctan \sqrt{\frac{P_{\mathrm{UP}}}{P_{\mathrm{UD}}}}$} \\
\hline 0 & {$\left[0, \frac{T_{\mathrm{sc}}}{4}\right)$} & $\theta_{\mathrm{e}}-\frac{\pi}{4}$ & $\frac{7 \pi}{4}-\theta_{\mathrm{e}}$ & $\frac{3 \pi}{4}-\theta_{\mathrm{e}}$ & $\theta_{\mathrm{e}}+\frac{3 \pi}{4}$ & $\theta_{\mathrm{O}}+\frac{\pi}{4}$ & $\frac{\pi}{4}-\theta_{\mathrm{o}}$ & $\frac{5 \pi}{4}-\theta_{\mathrm{o}}$ & $\theta_{\mathrm{O}}+\frac{5 \pi}{4}$ \\
\hline 1 & {$\left[\frac{T_{\mathrm{sc}}}{4}, \frac{2 T_{\mathrm{sc}}}{4}\right)$} & $\theta_{\mathrm{e}}-\frac{3 \pi}{4}$ & $\frac{5 \pi}{4}-\theta_{\mathrm{e}}$ & $\frac{\pi}{4}-\theta_{\mathrm{e}}$ & $\theta_{\mathrm{e}}+\frac{\pi}{4}$ & $\theta_{\mathrm{o}}+\frac{3 \pi}{4}$ & $\frac{3 \pi}{4}-\theta_{\mathrm{o}}$ & $\frac{7 \pi}{4}-\theta_{\mathrm{o}}$ & $\theta_{\mathrm{o}}+\frac{7 \pi}{4}$ \\
\hline 2 & {$\left[\frac{2 T_{\mathrm{sc}}}{4}, \frac{3 T_{\mathrm{sc}}}{4}\right)$} & $\theta_{\mathrm{e}}+\frac{3 \pi}{4}$ & $\frac{3 \pi}{4}-\theta_{\mathrm{e}}$ & $\frac{7 \pi}{4}-\theta_{\mathrm{e}}$ & $\theta_{\mathrm{e}}-\frac{\pi}{4}$ & $\theta_{\mathrm{o}}+\frac{5 \pi}{4}$ & $\frac{5 \pi}{4}-\theta_{\mathrm{o}}$ & $\frac{\pi}{4}-\theta_{\mathrm{o}}$ & $\theta_{\mathrm{o}}+\frac{\pi}{4}$ \\
\hline 3 & {$\left[\frac{3 T_{\mathrm{sc}}}{4}, T_{\mathrm{sc}}\right)$} & $\theta_{\mathrm{e}}+\frac{\pi}{4}$ & $\frac{\pi}{4}-\theta_{\mathrm{e}}$ & $\frac{5 \pi}{4}-\theta_{\mathrm{e}}$ & $\theta_{\mathrm{e}}-\frac{3 \pi}{4}$ & $\theta_{\mathrm{o}}+\frac{7 \pi}{4}$ & $\frac{7 \pi}{4}-\theta_{\mathrm{o}}$ & $\frac{3 \pi}{4}-\theta_{\mathrm{o}}$ & $\theta_{\mathrm{o}}+\frac{3 \pi}{4}$ \\
\hline
\end{tabular}

Tab. 2. The LUT for GTD-AltBOC-LU.

\begin{tabular}{|c|c|c|c|c|c|c|c|c|c|}
\hline \multicolumn{2}{|c|}{ Even/odd selector } & \multicolumn{4}{|c|}{ Even chip } & \multicolumn{4}{|c|}{ Odd chip } \\
\hline & $s_{\mathrm{LD}}$ & 1 & 1 & -1 & -1 & - & - & - & - \\
\hline & $s_{\mathrm{LP}}$ & - & - & - & - & 1 & 1 & -1 & -1 \\
\hline & $s_{\mathrm{UX}}$ & 1 & -1 & 1 & -1 & - & - & - & - \\
\hline & $s_{\mathrm{Uy}}$ & - & - & - & - & 1 & -1 & 1 & -1 \\
\hline \multicolumn{2}{|r|}{$t \bmod T_{\mathrm{sc}}$} & \multicolumn{8}{|c|}{$\theta$ such that $s(t)=\exp (\mathrm{j} \theta)$, where $\theta_{\mathrm{e}}=\arctan \frac{\sqrt{P_{\mathrm{Ux}}}-\sqrt{P_{\mathrm{LD}}}}{\sqrt{P_{\mathrm{Ux}}}+\sqrt{P_{\mathrm{LD}}}}$ and $\theta_{\mathrm{O}}=\arctan \frac{\sqrt{P_{\mathrm{Uy}}}-\sqrt{P_{\mathrm{LP}}}}{\sqrt{P_{\mathrm{Uy}}}+\sqrt{P_{\mathrm{LP}}}}$} \\
\hline 0 & {$\left[0, \frac{T_{\mathrm{sc}}}{4}\right)$} & $\theta_{\mathrm{e}}$ & $\frac{3 \pi}{2}-\theta_{\mathrm{e}}$ & $\frac{\pi}{2}-\theta_{\mathrm{e}}$ & $\pi+\theta_{\mathrm{e}}$ & $\frac{\pi}{2}+\theta_{\mathrm{o}}$ & $-\theta_{\mathrm{o}}$ & $\pi-\theta_{\mathrm{o}}$ & $\frac{3 \pi}{2}+\theta_{\mathrm{o}}$ \\
\hline 1 & {$\left[\frac{T_{\mathrm{sc}}}{4}, \frac{2 T_{\mathrm{sc}}}{4}\right)$} & $\pi-\theta_{\mathrm{e}}$ & $\frac{3 \pi}{2}+\theta_{\mathrm{e}}$ & $\frac{\pi}{2}+\theta_{\mathrm{e}}$ & $-\theta_{\mathrm{e}}$ & $\frac{3 \pi}{2}-\theta_{\mathrm{o}}$ & $\theta_{\mathrm{o}}$ & $\pi+\theta_{\mathrm{o}}$ & $\frac{\pi}{2}-\theta_{\mathrm{o}}$ \\
\hline 2 & {$\left[\frac{2 T_{\mathrm{sc}}}{4}, \frac{3 T_{\mathrm{sc}}}{4}\right)$} & $\pi+\theta_{\mathrm{e}}$ & $\frac{\pi}{2}-\theta_{\mathrm{e}}$ & $\frac{3 \pi}{2}-\theta_{\mathrm{e}}$ & $\theta_{\mathrm{e}}$ & $\frac{3 \pi}{2}+\theta_{\mathrm{o}}$ & $\pi-\theta_{\mathrm{o}}$ & $-\theta_{\mathrm{o}}$ & $\frac{\pi}{2}+\theta_{\mathrm{o}}$ \\
\hline 3 & {$\left[\frac{3 T_{\mathrm{sc}}}{4}, T_{\mathrm{sc}}\right)$} & $-\theta_{\mathrm{e}}$ & $\frac{\pi}{2}+\theta_{\mathrm{e}}$ & $\frac{3 \pi}{2}+\theta_{\mathrm{e}}$ & $\pi-\theta_{\mathrm{e}}$ & $\frac{\pi}{2}-\theta_{\mathrm{o}}$ & $\pi+\theta_{\mathrm{o}}$ & $\theta_{\mathrm{o}}$ & $\frac{3 \pi}{2}-\theta_{\mathrm{o}}$ \\
\hline
\end{tabular}

Tab. 3. The LUTs for GTD-AltBOC-DP and GTD-AltBOC-X.

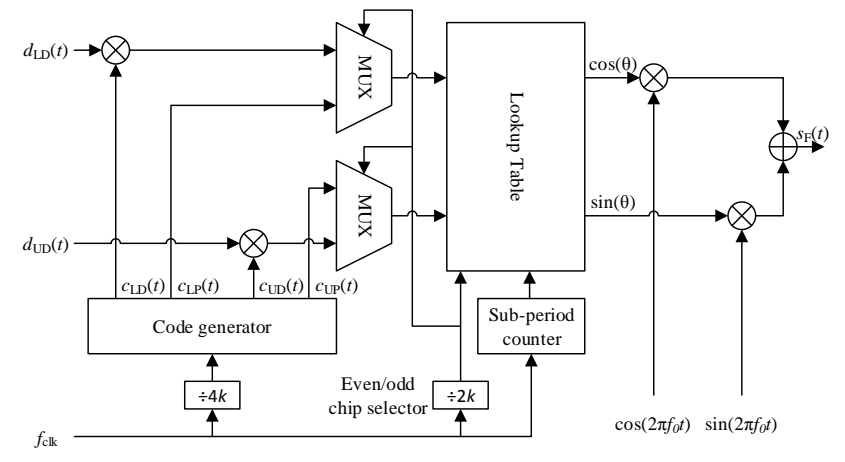

Fig. 3. Block diagram for GTD-AltBOC-X baseband signal generation using the LUT method.

signal generation. The four codes are generated by the code generator. The data-modulated codes and the pilot codes in the two sidebands are delivered to two 2-to-1 multiplexers. The outputs of the multiplexers and the even/odd chip selector are input to the LUT and used to determine the column address.

As shown in Tab. 2 and Tab. 3, time is first partitioned into subcarrier intervals of length $T_{\mathrm{sc}}$, each of which is further subdivided into 4 equal sub-periods. Therefore, the minimum required baseband clock rate is $f_{\text {clk }}=4 f_{\mathrm{sc}}$. Here, $k$ is the modulation index, which is defined as $k=2 n / m$, similar to the definition for $\operatorname{BOC}(m, n)$. The sub-period counter generates the row address in the LUT. The in-phase and quadrature components of the baseband signal are obtained from the LUT outputs. Then, these two components are modulated with the transmission carrier using a quadrature modulator to generate the RF signal to be transmitted.

As discussed above, the minimum required clock rate is $f_{\text {clk }}=4 f_{\text {sc }}$. For comparison, the minimum required clock rate for AltBOC modulation is $8 f_{\mathrm{sc}}$. The minimum required clock rate is a very important factor affecting the signal generation complexity in a satellite payload transmitter. Table 4 lists the minimum required clock rates for several existing methods. Note that the minimum required clock rate for ACE-BOC varies with the power allocation scheme; in Tab. 4, only PARs of $1: 1: 3: 3$ and 1:3:1:3 are considered for ACEBOC modulation. For GAltBOC modulation, the minimum required clock rate is $4 f_{\mathrm{sc}}$ for PARs of $1: \gamma^{2}: 1: \gamma^{2}$ and $8 f_{\mathrm{sc}}$ for other PARs, where $\gamma$ is a variable positive rational number. Compared with the signal generation hardware architecture 


\begin{tabular}{ccc}
\hline $\begin{array}{c}\text { Modulation } \\
\text { method }\end{array}$ & Power allocation & $\begin{array}{c}\text { Minimum required } \\
\text { clock rate }\end{array}$ \\
\hline AltBOC & $1: 1: 1: 1$ & $8 f_{\mathrm{sc}}$ \\
TD-AltBOC & $1: 1: 1: 1$ & $4 f_{\mathrm{sc}}$ \\
TMOC-QPSK & $1: 1: 1: 1$ & $4 f_{\mathrm{sc}}$ \\
& $1: 3: 1: 3$ & $12 f_{\mathrm{sc}}$ \\
ACE-BOC & $1: 1: 3: 3$ & $24 f_{\mathrm{sc}}$ \\
& $1: \gamma^{2}: 1: \gamma^{2}$ & $4 f_{\mathrm{sc}}$ \\
GAltBOC & Others & $8 f_{\mathrm{sc}}$ \\
GTD-AltBOC & Arbitrary & $4 f_{\mathrm{sc}}$ \\
\hline
\end{tabular}

Tab. 4. The minimum required clock rates for the proposed method and several existing methods.

for non-time-division modulation, a time-division method requires two additional multiplexers and an additional timeswitching circuit, but the minimum required clock rate is significantly reduced, which results in an obvious decrease in implementation complexity for signal generation in a satellite payload.

\section{Receiving Modes and Complexity Analysis}

For a dual-frequency constant-envelope-modulated signal, the entire composite signal and any individual signal component can be processed using similar techniques. The common tracking architecture for a GNSS receiver is shown in Fig. 4. For different reference baseband signals, a coherent dual-frequency constant envelope signal has at least three typical receiving modes [12], [15]:

- The entire composite signal is correlated with the fullband received signal using a LUT; this mode is referred to as full-band matched receiving (FMR).

- The reference baseband signal for the desired signal component is generated associated with the appropriate complex subcarrier; this mode is referred to as full-band independent correlation (FIC).

- The signals in each sideband are translated from their center frequencies to the baseband and are then processed as two QPSK signals, and the reference baseband signal is generated independently of the subcarriers; this mode is referred to as sideband translation (SBT).

The locally generated carriers have a frequency of $f_{0}$ for the FMR and FIC modes and frequencies of $f_{0}-f_{\mathrm{sc}}, f_{0}+f_{\mathrm{sc}}$, or both for the SBT mode. For the FIC and SBT receiving modes, several combinations of signal components are possible; these combinations may be coherent or non-coherent and may involve different selections of the data and pilot components and of the lower- and upper-sideband components [15].

Note that the ambiguity of the navigation message bits should be resolved if the tracking channel contains a data component. For the FMR receiving mode, four parallel correlators are required to process the four possible hypotheses

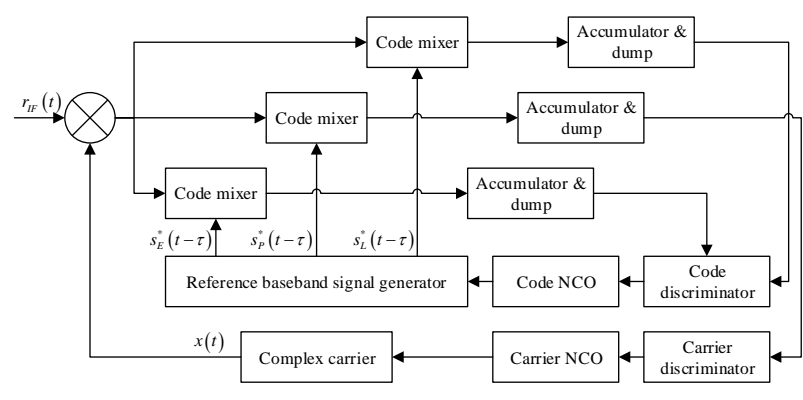

Fig. 4. The common tracking architecture for a GNSS receiver.

\begin{tabular}{ccc}
\hline $\begin{array}{c}\text { Receiving } \\
\text { mode }\end{array}$ & $\begin{array}{c}\text { Signal component } \\
\text { of interest }\end{array}$ & $\begin{array}{c}\text { Reference } \\
\text { baseband signal }\end{array}$ \\
\hline FMR & $s$ & $\exp (\mathrm{j} \theta(t-\tau))$ \\
\hline FIC & $s_{\mathrm{p}}$ & $\begin{array}{c}s_{\mathrm{LP}}(t-\tau) S C_{\mathrm{L}}(t-\tau) \\
\& s_{\mathrm{UP}}(t-\tau) S C_{\mathrm{U}}(t-\tau)\end{array}$ \\
\hline SBT & $s_{\mathrm{LP}}$ & $s_{\mathrm{LP}}(t-\tau)$ \\
\hline
\end{tabular}

Tab. 5. The local reference baseband signals of interest for the three receiving modes.

regarding the navigation message bits. The numbers of additional parallel correlators for all the considered modulations are the same. Therefore, for comparison with other existing methods, it is sufficient to analyze only one correlator. For the FIC and SBT modes, to avoid ambiguity of the navigation message bits, we consider only the pilot components in this paper. The combinations of interest are listed in Tab. 5, where $\tau$ denotes the delay and $\theta$ takes the values listed in Tab. 2 and Tab. 3.

For a hardware receiver, the hardware complexity and power consumption are two important indicators of performance. In general, power consumption and hardware complexity are positively correlated; a higher hardware complexity is associated with higher power consumption. Therefore, this section primarily focuses on the hardware complexity for each receiving mode.

In the receiver architecture depicted in Fig. 4, the reference baseband signal generator, the code mixers and the accumulators are the three core components that differ among the cases of different types of coherent dual-frequency constant envelope signals. Therefore, to compare the complexities of the different methods, we mainly analyze the complexities of these three components.

\subsection{Storage Complexity for the FMR Mode}

To illustrate the complexity analysis process in detail, we consider GTD-AltBOC(15,10,1:3:1:3) modulation as an example. The complexity analysis is illustrated by means of a schematic diagram in Fig. 5.

As seen in Fig. 2, the real and imaginary parts of the composite signal take four values, $\{ \pm 0.2588, \pm 0.9659\}$. Obviously, these are floating point values, which are complex to 


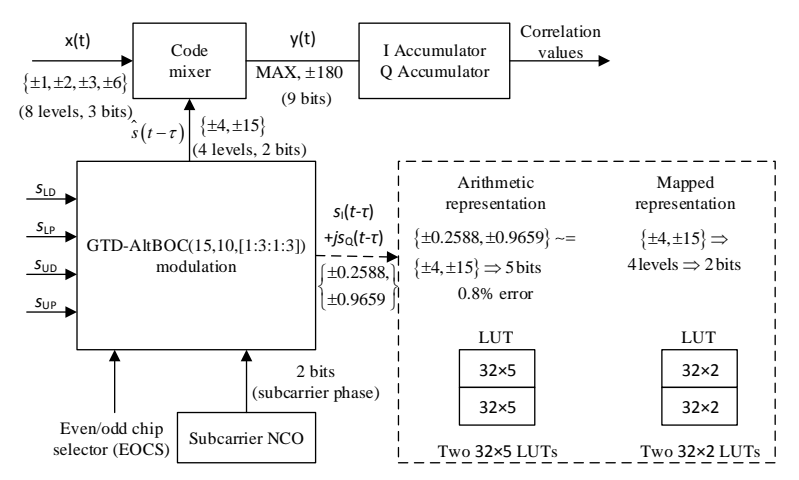

Fig. 5. Complexity analysis of an FMR receiver for GTDAltBOC(15,10,1:3:1:3)-modulated signals

process in hardware. Consequently, in hardware implementations, floating point values are often replaced with scaled integers to reduce this complexity. We adopt the scaled integers $\{ \pm 4, \pm 15\}$ to represent the four floating point values $\{ \pm 0.2588, \pm 0.9659\}$, with an error of $0.8 \%$.

For the FMR receiving mode, the reference baseband signal is generated using the LUT method, which is well suited for hardware implementation. The real and imaginary parts of the output can be represented using approximate integer arithmetic. Five bits are required to represent the four floating point values with the scaled integers $\{ \pm 4, \pm 15\}$. Consequently, the arithmetic representation of the LUT method requires two $32 \times 5$ LUTs (one for I and one for Q). In general, the next stage of reference signal generation is a code mixer, which is also implemented as a LUT. As part of the LUT address that is input into the code mixer, the reference signal does not require a true-value representation. Therefore, we can simply represent the reference signal by means of a mapping representation [5]. For the four-level reference signal, a 2-bit representation is sufficient. As a result, the mapping representation for the reference signal generator requires only two $32 \times 2$ LUTs (one for I and one for Q).

The code mixer is also implemented as a LUT. The reference baseband signal and the received baseband signal are the two inputs to the code mixer. As previously analyzed, the reference baseband signal is represented using 2 bits. The received baseband signal can generally be represented as $\{ \pm 1, \pm 2, \pm 3, \pm 6\}$, a mapped representation of which requires 3 bits. The 3-bit encoding of $\mathrm{x}(\mathrm{t})$ and the 2-bit encoding of $\hat{s}(t-\tau)$ then serve as the inputs to the code mixer. The maximum outputs of the code mixer are \pm 180 , which require 9 bits for representation. Therefore, the LUT implementation of the code mixer requires four $32 \times 9$ LUTs.

Table 6 summarizes the storage space required for the reference signal generator and code mixer for an FMR receiver. Obviously, for each PAR, the GTD-AltBOC method requires the lowest complexity of both the reference signal generator and the code mixer. Such reductions in complexity are important for receiver design, especially for portable receivers. Note that for the PAR of 1:1:1:1, the GTD-AltBOC

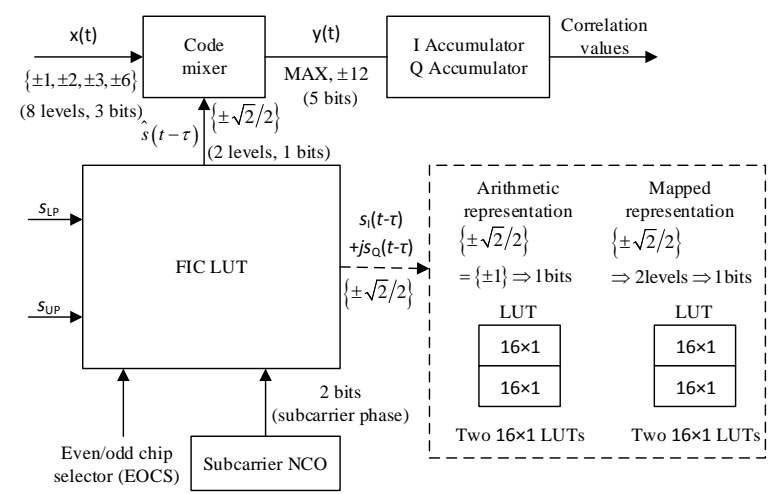

Fig. 6. Complexity analysis of an FIC receiver for GTDAltBOC(15,10,1:3:1:3)-modulated signals.

signal and the TD-AltBOC signal are both two-level signals and have similar signal structures; therefore, these two methods have the same storage complexities, as shown in Tab. 6.

\subsection{Storage Complexity for the FIC Mode}

An analysis similar to that for the FMR receiving mode can be conducted for an FIC receiver. The corresponding complexity analysis is illustrated by means of a schematic diagram in Fig. 6, and the resulting comparison of the proposed GTD-AltBOC method with existing methods is presented in Tab. 7. Again, GTD-AltBOC modulation imposes the lowest complexity requirements for both the reference signal generator and the code mixer.

\subsection{Storage Complexity for the SBT Mode}

For the SBT receiving mode, AltBOC, TD-AltBOC, TMOC-QPSK, ACE-BOC, GAltBOC and GTD-AltBOC signals can all be processed in the same way as a BPSK signal. Therefore, all of these methods have similar storage complexities, aside from the fact that the time-division methods require an additional time-switching circuit, which has little impact on the receiver storage complexity.

\subsection{Computational Complexity Analysis}

The computational complexity can be evaluated by the times of multiplications, additions/subtractions, and bit width. In general, the output of the reference signal generator is produced using a LUT method, which does not require any multiplications or additions/subtractions. By contrast, the code mixer multiplies the local reference signal by the received signal, which is a multiplication operation involving two complex signals. If a direct computation method were to be used, the code mixer would perform four multiplications and two additions/subtractions. However, as mentioned above, a LUT implementation is also used for the code mixer in the approach considered in this paper, meaning that the four multiplications are implemented using four LUTs. Therefore, only two addition/subtraction operations must be considered for the code mixer. Finally, the accumulators must accumulate a large number of samples, a process that depends on the sampling rate, the coherent integration time, and bit width. 


\begin{tabular}{|c|c|c|c|c|}
\hline PAR & $\begin{array}{l}\text { Modulation } \\
\text { method }\end{array}$ & Representation of $\hat{s}(t-\tau)$ & $\begin{array}{c}\text { Reference } \\
\text { signal generator }\end{array}$ & Code mixer \\
\hline \multirow{3}{*}{$1: 1: 1: 1$} & AltBOC & $\begin{array}{l}\{0, \pm 0.707, \pm 1\} \sim=\{0, \pm 5, \pm 7\} \\
\quad \Rightarrow 4 \text { bits }(5 \text { levels, } 3 \text { bits })\end{array}$ & Two $128 \times 3$ LUTs & Four $64 \times 8$ LUTs \\
\hline & TD-AltBOC & $\{ \pm 1\} \sim=\{ \pm 1\} \Rightarrow 1$ bit (2 levels, 1 bit $)$ & Two $32 \times 1$ LUTs & Four $16 \times 5$ LUTs \\
\hline & GTD-AltBOC & $\{ \pm 1\} \sim=\{ \pm 1\} \Rightarrow 1$ bit (2 levels, 1 bit $)$ & Two $32 \times 1$ LUTs & Four $16 \times 5$ LUTs \\
\hline \multirow{3}{*}{$1: 3: 1: 3$} & ACE-BOC & $\begin{array}{c}\{0, \pm 0.5, \pm 0.866, \pm 1\} \sim=\{0, \pm 7, \pm 12, \pm 14\} \\
\Rightarrow 5 \text { bits ( } 7 \text { levels, } 3 \text { bits })\end{array}$ & Two $192 \times 3$ LUTs & Four $64 \times 9$ LUTs \\
\hline & GAltBOC & $\begin{array}{c}\{0, \pm 0.5, \pm 0.866, \pm 1\} \sim=\{0, \pm 7, \pm 12, \pm 14\} \\
\Rightarrow 5 \text { bits ( } 7 \text { levels, } 3 \text { bits })\end{array}$ & Two $64 \times 3$ LUTs & Four $64 \times 9$ LUTs \\
\hline & GTD-AltBOC & $\begin{array}{l}\{ \pm 0.2588, \pm 0.9659\} \sim=\{ \pm 4, \pm 15\} \\
\Rightarrow 5 \text { bits }(4 \text { levels, } 2 \text { bits })\end{array}$ & Two $32 \times 2$ LUTs & Four $32 \times 9$ LUTs \\
\hline \multirow{4}{*}{$1: 1: 3: 3$} & ACE-BOC & $\begin{array}{c}\{ \pm 0.2588, \pm 0.7071, \pm 0.9659\} \sim=\{ \pm 4, \pm 11, \pm 15\} \\
\quad \Rightarrow 5 \text { bits }(6 \text { levels, } 3 \text { bits })\end{array}$ & Two $384 \times 3$ LUTs & Four $64 \times 9$ LUTs \\
\hline & GAltBOC & $\left\{\begin{array}{l} \pm 0.063, \pm 0.3455, \pm 0.4193 \\
\pm 0.6611, \pm 0.7503, \pm 0.9079 \\
\pm 0.9384, \pm 0.9980\end{array}\right\} \sim=\left\{\begin{array}{l} \pm 4, \pm 22, \pm 27 \\
\pm 42, \pm 48, \pm 58 \\
\pm 60, \pm 64\end{array}\right\}$ & Two $128 \times 4$ LUTs & Four $128 \times 11$ LUTs \\
\hline & & $\Rightarrow 8$ bits ( 16 levels, 4 bits $)$ & & \\
\hline & GTD-AltBOC & $\begin{array}{l}\{ \pm 0.2588, \pm 0.9659\} \sim=\{ \pm 4, \pm 15\} \\
\quad \Rightarrow 5 \text { bits }(4 \text { levels, } 2 \text { bits })\end{array}$ & Two $32 \times 2$ LUTs & Four $32 \times 9$ LUTs \\
\hline
\end{tabular}

Tab. 6. The storage space required for the reference signal generator and code mixer for an FMR receiver.

\begin{tabular}{|c|c|c|c|c|}
\hline PAR & $\begin{array}{l}\text { Modulation } \\
\text { method }\end{array}$ & Representation of $\hat{s}(t-\tau)$ & $\begin{array}{c}\text { Reference } \\
\text { signal generator }\end{array}$ & Code mixer \\
\hline \multirow{3}{*}{$1: 1: 1: 1$} & AltBOC & $\begin{array}{c}\{0, \pm 0.433, \pm 0.933\} \sim=\{0, \pm 13, \pm 28\} \\
\Rightarrow 6 \text { bits }(5 \text { levels, } 3 \text { bits })\end{array}$ & Two $32 \times 3$ LUTs & Four $64 \times 10$ LUTs \\
\hline & TD-AltBOC & $\{ \pm 1\} \sim=\{ \pm 1\} \Rightarrow 1$ bit (2 levels, 1 bit $)$ & Two $16 \times 1$ LUTs & Four $16 \times 5$ LUTs \\
\hline & GTD-AltBOC & $\{ \pm 1\} \sim=\{ \pm 1\} \Rightarrow 1$ bit (2 levels, 1 bit $)$ & Two $16 \times 1$ LUTs & Four $16 \times 5$ LUTs \\
\hline \multirow{3}{*}{$1: 3: 1: 3$} & ACE-BOC & $\begin{array}{c}\{0, \pm 0.433, \pm 0.933\} \sim=\{0, \pm 13, \pm 28\} \\
\Rightarrow 6 \text { bits }(5 \text { levels, } 3 \text { bits })\end{array}$ & Two $48 \times 3$ LUTs & Four $64 \times 10$ LUTs \\
\hline & GAltBOC & $\{ \pm 1\} \sim=\{ \pm 1\} \Rightarrow 1$ bit (2 levels, 1 bit $)$ & Two $16 \times 1$ LUTs & Four $16 \times 5$ LUTs \\
\hline & GTD-AltBOC & $\{ \pm 1\} \sim=\{ \pm 1\} \Rightarrow 1$ bit (2 levels, 1 bit $)$ & Two $16 \times 1$ LUTs & Four $16 \times 5$ LUTs \\
\hline \multirow{3}{*}{$1: 1: 3: 3$} & ACE-BOC & $\begin{array}{c}\left\{\begin{array}{l}0, \pm 0.1294, \pm 0.3536 \\
\pm 0.483, \pm 0.8365\end{array}\right\} \sim=\left\{\begin{array}{l}0, \pm 6, \pm 17 \\
\pm 23, \pm 40\end{array}\right\} \\
\Rightarrow 7 \text { bits }(9 \text { levels, } 4 \text { bits })\end{array}$ & Two $96 \times 4$ LUTs & Four $128 \times 10$ LUTs \\
\hline & GAltBOC & $\begin{array}{c}\left\{\begin{array}{l} \pm 0.0929, \pm 0.2243 \\
\pm 0.3467, \pm 0.837\end{array}\right\} \sim=\left\{\begin{array}{l} \pm 5, \pm 12 \\
\pm 19, \pm 45\end{array}\right\} \\
\Rightarrow 7 \text { bits ( } 8 \text { levels, } 3 \text { bits })\end{array}$ & Two $32 \times 3$ LUTs & Four $64 \times 11$ LUTs \\
\hline & GTD-AltBOC & $\begin{array}{l}\{ \pm 0.5, \pm 0.866\} \sim=\{ \pm 7, \pm 12\} \\
\quad \Rightarrow 5 \text { bits }(4 \text { levels, } 2 \text { bits })\end{array}$ & Two $16 \times 2$ LUTs & Four $32 \times 9$ LUTs \\
\hline
\end{tabular}

Tab. 7. The storage space required for the reference signal generator and code mixer for an FIC receiver.

For both the FMR and FIC modes, given the same sampling rate and coherent integration time, the number of samples collected in a single coherent integration time window will be the same for an AltBOC, TMOC-QPSK, ACE-BOC, GAltBOC or GTD-AltBOC receiver. Hence, for all of these methods, the same number of accumulations will be performed per coherent integration time window. Note that if the sampling rate is very high due to a wide receiving bandwidth, then the number of samples per integration period will also be very large. Therefore, the bit width of the code mixer LUTs will strongly affect the computational complexity of the code mixer and the accumulators. For the example of GTD-AltBOC $(15,10,1: 3: 1: 3)$ in the FMR receiving mode, the code mixer needs two 9-bit width additions/subtractions, 
the 9-bit width outputs are also the inputs to the I and Q accumulators. The bit width of the accumulator outputs will reach $9+\log _{2}$ (samples), where samples denotes the number of samples collected in one coherent integration time window. Because the number of samples is the same for all considered methods, the computational complexity predominantly depends on the bit width of the code mixer.

The last columns of Tab. 6 and Tab. 7 list the storage space required for the code mixer. The number after the multiplication sign (" $x$ ") represents the bit width of each LUT cell, which is also the bit width of the code mixer outputs. From these tables, we can conclude that the computational complexity of the proposed GTDAltBOC method is also reduced compared with the existing methods for the FMR and FIC receiving modes.

For the SBT receiving mode, as in the case of the storage complexity, all of the considered methods have similar computational complexities.

\section{Simulations and Performance Anal- ysis}

In this section, simulations of PSDs, cross-correlation functions, multiplexing efficiencies and code-tracking performance are reported. Parameters similar to those of the Galileo E5 signal were used in the simulations: a code rate of $R_{\mathrm{c}}=10.23 \mathrm{MHz}$ and a subcarrier frequency of $f_{\mathrm{sc}}=15 \times 1.023 \mathrm{MHz}$ [1]. The PRN code length was set to 5115 for time-division methods and 10230 for non-time-division methods. Note that TMOC-QPSK is a special cases of GTD-AltBOC; thus, we did not include this method in the simulations. As previously considered with respect to ACE-BOC [11], [12] and GAltBOC [14], two PARs of 1:1:3:3 and 1:3:1:3 were considered in the simulations. All of the simulations reported in this section were performed using the MATLAB platform.

\subsection{Power Spectral Density (PSD)}

A binary complex subcarrier essentially consists of a sine-phase subcarrier and a cosine-phase subcarrier, as expressed in (1). The PSD of GTD-AltBOC can be obtained by combining the PSDs of sine-BOC and cosine$\mathrm{BOC}$, which are given in reference [5]. When $k=$ $2 f_{\mathrm{sc}} / f_{\mathrm{c}}$ is odd, the normalized PSD of GTD-AltBOC is

$$
G_{\mathrm{GTD}}^{o}(f)=\frac{2 R_{\mathrm{c}}}{(\pi f)^{2}} \frac{\cos ^{2}\left(\frac{\pi f}{R_{\mathrm{c}}}\right) \sin ^{2}\left(\frac{\pi f}{4 f_{\mathrm{sc}}}\right)}{\cos ^{2}\left(\frac{\pi f}{2 f_{\mathrm{sc}}}\right)} P(f) .
$$

When $\mathrm{k}$ is even, the normalized PSD of GTD-AltBOC is

$$
G_{\mathrm{GTD}}^{e}(f)=\frac{2 R_{\mathrm{c}}}{(\pi f)^{2}} \frac{\sin ^{2}\left(\frac{\pi f}{R_{\mathrm{c}}}\right) \sin ^{2}\left(\frac{\pi f}{4 f_{\mathrm{sc}}}\right)}{\cos ^{2}\left(\frac{\pi f}{2 f_{\mathrm{sc}}}\right)} P(f) .
$$

Here, $P(f)$ is the power allocation factor, which is defined as

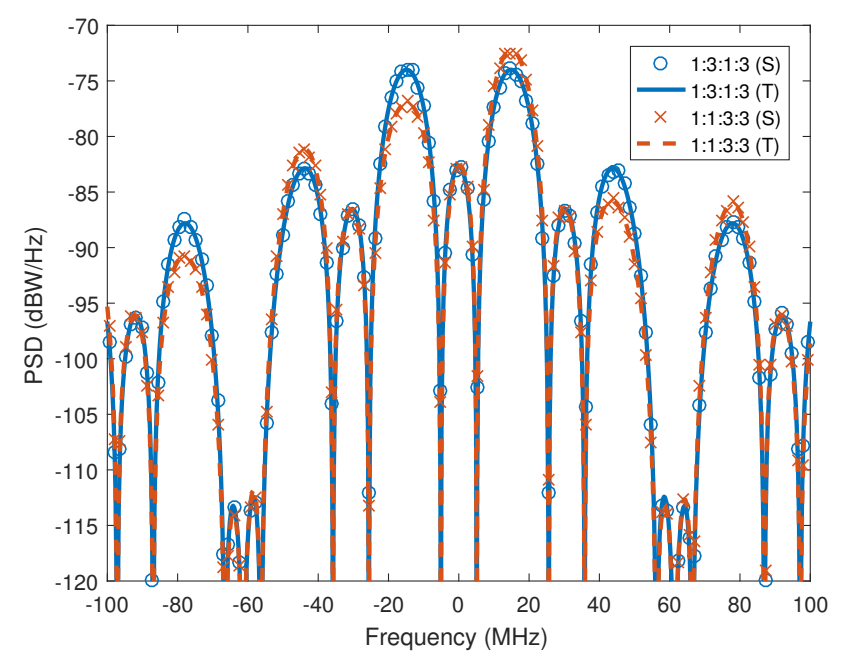

Fig. 7. The simulated and theoretical PSDs of GTD-AltBOC.

$$
P(f)=1+\frac{P_{\mathrm{UD}}+P_{\mathrm{UP}}-P_{\mathrm{LD}}-P_{\mathrm{LP}}}{P_{\mathrm{UD}}+P_{\mathrm{UP}}+P_{\mathrm{LD}}+P_{\mathrm{LP}}} \sin \left(\frac{\pi f}{2 f_{\mathrm{sc}}}\right) .
$$

Obviously, $P(f)=1$ for the case of equal power allocation among all four signal components, for which (10) and (11) degenerate to the PSD expressions for TD-AltBOC and TMOC-QPSK, respectively.

For the presented simulations, the theoretical PSD of GTD-AltBOC was calculated for each PAR in accordance with (10)-(12). The simulated PSD was obtained by generating a composite signal with random spread codes at a complex sampling rate of $300 \mathrm{MHz}$ and averaging the powers from 10000 repeated FFT computations; then, the result was improved by means of a correction factor to compensate for the sampling effect [16].

Figure 7 shows the simulated and theoretical PSDs of GTD-AltBOC for PARs of 1:3:1:3 and 1:1:3:3. The notations "S" and "T" indicate "simulated" and "theoretical", respectively. We find that the simulated PSDs are consistent with the theoretical PSDs, thereby verifying the correctness of the theoretical PSD expression. The peaks at $\pm f_{\text {sc }}$ reflect the power relationship between the two sidebands. As seen from Fig. 7, the values of the two peaks are equal for the PAR of $1: 3: 1: 3$, whereas the peak at $f_{\text {sc }}$ is 4.77 $\mathrm{dB}$ higher than that at $-f_{\mathrm{sc}}$ for the PAR of $1: 1: 3: 3$; these results exactly correspond to the power ratios between the two sidebands for the two PARs. The shapes of the PSDs illustrate that the four signal components are indeed modulated at the correct frequencies via GTD-AltBOC modulation.

\subsection{Cross-correlation Function}

The cross-correlation function reflects whether the signal components are combined correctly with the desired modulation, power and phase. Similar to the case of TMOC-QPSK, the cross-correlation functions were simulated for each signal component and the composite signal. Figure 8 shows the simulation architecture used to 


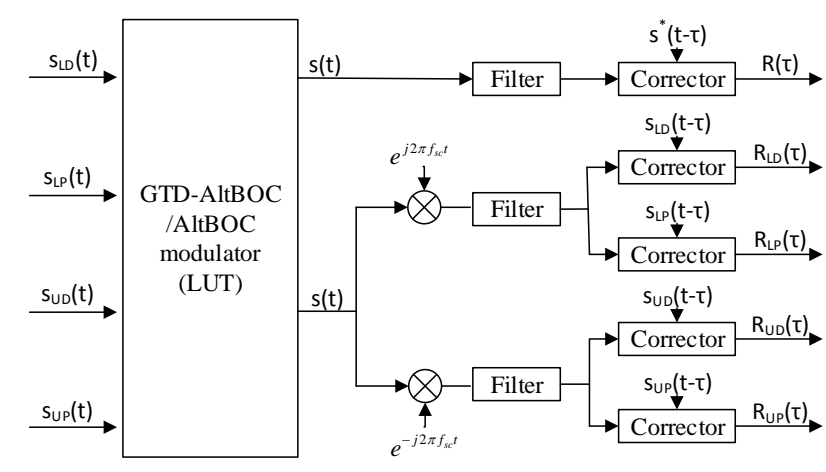

Fig. 8. The simulation architecture used to obtain the crosscorrelation functions of AltBOC and GTD-AltBOC signals.

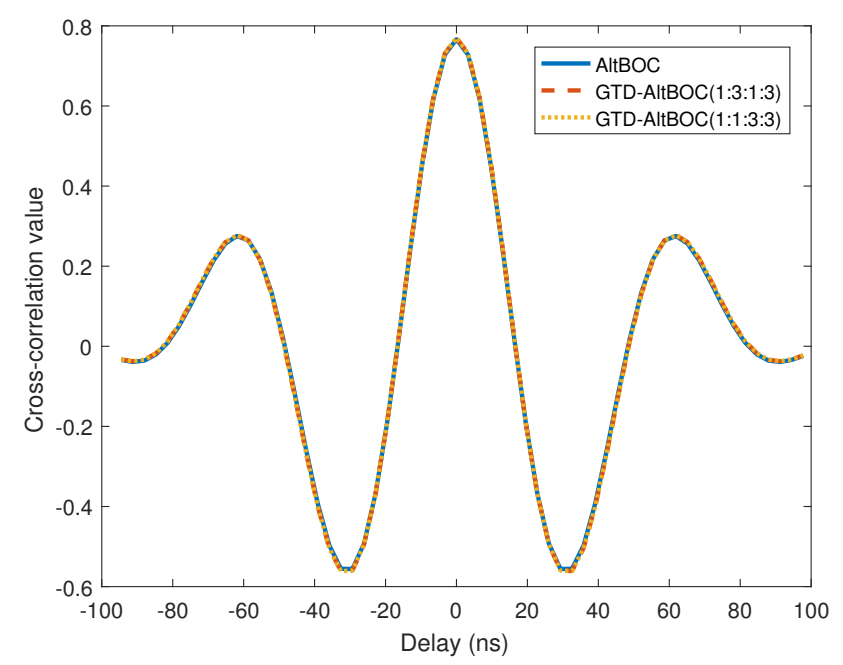

Fig. 9. The cross-correlation functions of AltBOC and GTDAltBOC signals.

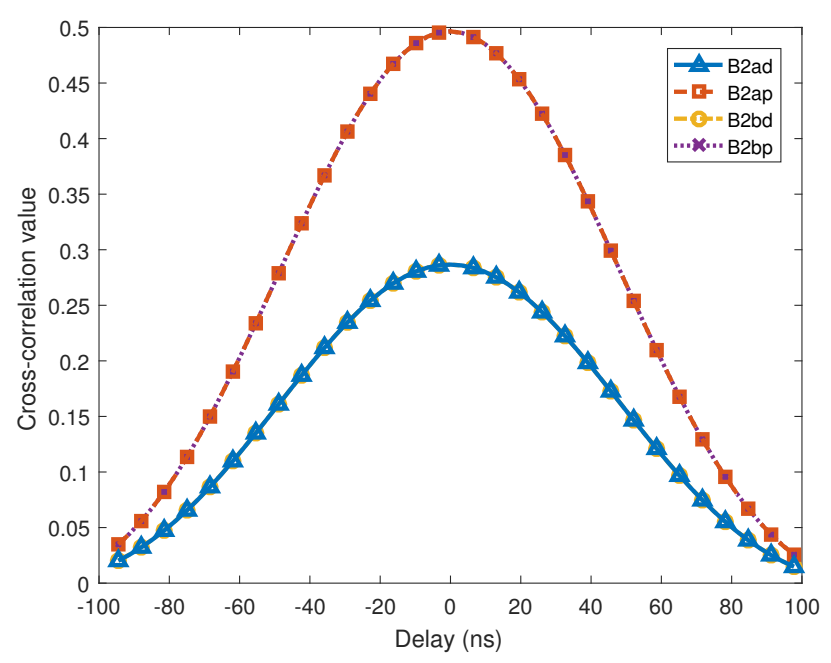

Fig. 10. The cross-correlation functions of the four signal components of a GTD-AltBOC(15,10,1:3:1:3) signal.

obtain the cross-correlation functions of AltBOC and GTDAltBOC signals. The four signal components $s_{\mathrm{LD}}(t), s_{\mathrm{LP}}(t)$,
$s_{\mathrm{UD}}(t)$, and $s_{\mathrm{UP}}(t)$ were generated with random spread codes. A complex sampling rate of $300 \mathrm{MHz}$, a filter bandwidth of $51.15 \mathrm{MHz}$ for composite signal processing, and a filter bandwidth of $20.46 \mathrm{MHz}$ for the processing of each signal component were adopted, and the cross-correlation functions were averaged over 1000 repetitions. $s_{\mathrm{LD}}(t-\tau), s_{\mathrm{LP}}(t-\tau)$, $s_{\mathrm{UD}}(t-\tau), s_{\mathrm{UP}}(t-\tau)$ and $s(t-\tau)$ denote the local replicas of the four signal components and the composite signal. $s^{*}(t-\tau)$ denotes the conjugate of $s(t)$ with delay $\tau . R_{\mathrm{LD}}(\tau)$, $R_{\mathrm{LP}}(\tau), R_{\mathrm{UD}}(\tau), R_{\mathrm{UP}}(\tau)$ and $R(\tau)$ are the cross-correlation functions corresponding to the four signal components and the composite signal.

Figure 9 shows the simulated cross-correlation functions of the composite signals for AltBOC and GTD-AltBOC, where PARs of 1:3:1:3 and 1:1:3:3 are considered for GTDAltBOC. The three cross-correlation functions are essentially identical, which implies that GTD-AltBOC(15,10,1:3:1:3) and GTD-AltBOC $(15,10,1: 1: 3: 3)$ can achieve the same ranging performance as $\operatorname{AltBOC}(15,10)$ in the FMR receiving mode.

Figure 10 shows the cross-correlation functions of the four signal components for a GTD-AltBOC(15,10,1:3:1:3) signal. The main peaks for the four signal components lie at $\left|R_{\mathrm{LD}}(0)\right|=\left|R_{\mathrm{UD}}(0)\right|=0.2866$ and $\left|R_{\mathrm{LP}}(0)\right|=\left|R_{\mathrm{UP}}(0)\right|=0.4965$. The simulated PAR is $\left|R_{\mathrm{LD}}(0)\right|^{2}:\left|R_{\mathrm{LP}}(0)\right|^{2}:\left|R_{\mathrm{UD}}(0)\right|^{2}:\left|R_{\mathrm{UP}}(0)\right|^{2}=$ 1:3.0000:1:3.0000, consistent with the designed PAR. For GTD-AltBOC $(15,10,1: 1: 3: 3)$ modulation, conclusions similar to those for GTD-AltBOC $(15,10,1: 3: 1: 3)$ can be drawn; to avoid unnecessary repetition, the details are not presented.

The cross-correlation functions shown in Fig. 9 and Fig. 10 demonstrate that the GTD-AltBOC method can successfully modulate the four signal components to the assigned frequency with the desired PAR.

\subsection{Multiplexing Efficiency}

The multiplexing efficiency is a power ratio defined as the sum of the powers of the required signal components divided by the power of the composite signal, as follows [16]:

$$
\eta=\frac{\sum_{n=1}^{4}\left|\operatorname{corr}_{n}\right|^{2}}{P_{\mathrm{T}}}
$$

where $P_{n}=\left|\operatorname{corr}_{n}\right|^{2}$ is the correlation power of the n-th required signal component, as measured by a correlation receiver matched to that signal component, and $P_{\mathrm{T}}$ is the total power of the composite signal $\left(P_{\mathrm{T}}=1\right.$ in the simulation). In the FMR receiving mode, the total composite signal is processed, meaning that the correlation power of the required component is equal to the power of the composite signal. Therefore, all of the modulation methods have the same multiplexing efficiency of $\eta=100 \%$. In the FIC and SBT receiving modes, the multiplexing efficiency depends on the PAR. In the simulations, only the FIC and SBT modes were considered, for two different PAR scenarios, which have also been discussed by Yao [12] and Yan [14]: I. The data 

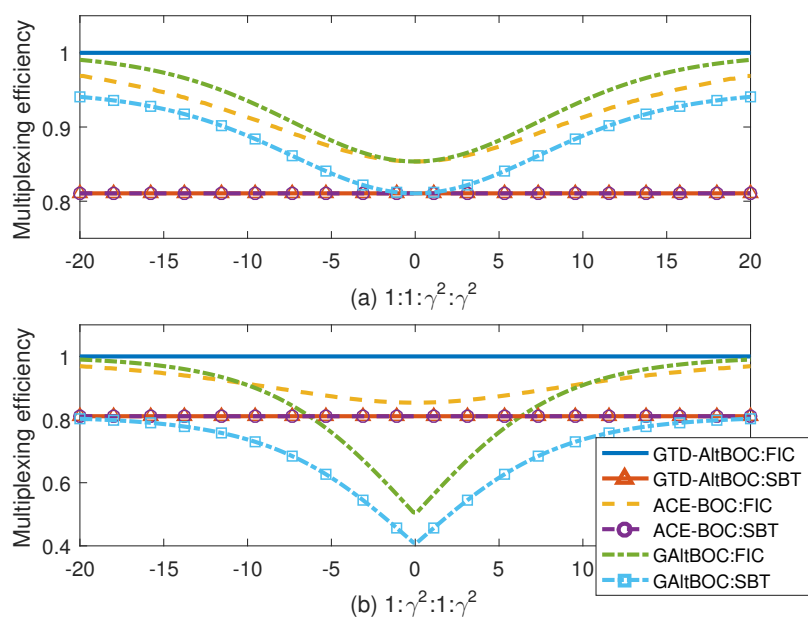

Fig. 11. Comparison of the multiplexing efficiencies of GTDAltBOC, ACE-BOC and GAltBOC.

component and the pilot component have equal powers, but the power ratio between the lower and upper sidebands is $1: \gamma^{2}$, where $\gamma$ is a variable positive real number; in other words, the PAR is $1: 1: \gamma^{2}: \gamma^{2}$. II. More power is preferentially allocated to the pilot component, but the power ratio between the lower and upper sidebands is $1: 1$; in other words, the PAR is $1: \gamma^{2}: 1: \gamma^{2}$. Note that the PAR must be $1: 1: 1: 1$ for both AltBOC and TD-AltBOC; their multiplexing efficiencies are not shown in the following figure, but they are listed in Tab. 8.

The multiplexing efficiencies of GTD-AltBOC, ACEBOC and GAltBOC for different PARs and different receiving modes are shown in Fig. 11. The $\mathrm{x}$ axis represents the $\operatorname{logarithm}$ of the power ratio, which is $20 \log (\gamma)$. As seen from Fig. 11, for any modulation method, the FIC receiving mode always has a higher multiplexing efficiency than the SBT mode.

For the FIC receiving mode, the multiplexing efficiency of GTD-AltBOC is $100 \%$. By contrast, because of the existence of additional inter-product components in the ACE$\mathrm{BOC}$ and GAltBOC signals, the multiplexing efficiencies of ACE-BOC and GAltBOC are both less than $100 \%$. As seen from Fig. 11, the proposed GTD-AltBOC modulation method has the highest multiplexing efficiency compared with ACEBOC and GAltBOC for both PAR scenarios. For certain values of $\gamma$, the multiplexing efficiency of GAltBOC for a PAR of $1: \gamma^{2}: 1: \gamma^{2}$ is particularly low.

For the SBT receiving mode, regardless of which PAR is chosen, the multiplexing efficiencies of GTD-AltBOC and ACE-BOC remain constant at $81.06 \%$. By contrast, GAlt$\mathrm{BOC}$ has a higher multiplexing efficiency than that of either GTD-AltBOC or ACE-BOC for a PAR of $1: 1: \gamma^{2}: \gamma^{2}$ and a lower multiplexing efficiency than that of either GTDAltBOC or ACE-BOC for a PAR of $1: \gamma^{2}: 1: \gamma^{2}$.

As specific examples, the multiplexing efficiencies for AltBOC, TD-AltBOC, GTD-AltBOC, ACE-BOC and GAltBOC are provided for PARs of 1:1:1:1, 1:3:1:3 and 1:1:3:3 in Tab. 8, where 'GTD-' denotes the proposed GTD-AltBOC method.

\subsection{Code-Tracking Performance}

The code-tracking error is an important indicator of tracking performance. For coherent early-late processing (CELP), the lower bound of the code-tracking error with additive white Gaussian noise (AWGN) [17] is

$$
\sigma_{\mathrm{LB}, \text { white }}^{2}=\frac{B_{\mathrm{L}}\left(1-0.5 B_{\mathrm{L}} T_{\mathrm{i}}\right)}{(2 \pi)^{2} \frac{C}{N_{0}} \int_{-B_{\mathrm{r}} / 2}^{B_{\mathrm{r}} / 2} f^{2} \mathrm{G}(f) d f}
$$

where $B_{\mathrm{L}}$ is the code-tracking loop bandwidth, $T_{\mathrm{i}}$ is the integration time, $C / N_{0}$ is the carrier-to-noise-density ratio (CNR), $B_{\mathrm{r}}$ is the pre-filtering bandwidth of the receiver, and $G(f)$ is the PSD of the required signal. In the simulations reported here, the receiving bandwidth was $51.15 \mathrm{MHz}$ for the FMP and FIC receiving modes and $20.46 \mathrm{MHz}$ for the SBT receiving mode, the integration time was $1 \mathrm{~ms}$, and the code-tracking loop bandwidth was $1 \mathrm{~Hz}$ [18].

If the influence of bandlimiting is not considered, for the same receiving mode and receiver parameters, the codetracking performance depends on the PSD and the effective power of the required components. As an example, we consider a comparison between GAltBOC with a PAR of 1:3:1:3 and AltBOC in the FIC receiving mode. The shape of the PSD is similar for both methods. Although the multiplexing efficiency of GAltBOC is $0.56 \mathrm{~dB}$ lower than that of AltBOC, as shown in Tab. 8, the pilot power proportion for GAltBOC is 0.75 , which is $1.76 \mathrm{~dB}$ greater than that for AltBOC. Therefore, GAltBOC still has a $1.2 \mathrm{~dB}$ improvement in code-tracking performance over AltBOC. Because of their similar PSDs and higher multiplexing efficiencies, GTDAltBOC,TD-AltBOC and ACE-BOC will certainly achieve even greater improvements than GAltBOC in comparison with AltBOC.

For the case of a limited bandwidth, Fig. 12 shows the simulated code-tracking errors of GTD-AltBOC, AltBOC and TD-AltBOC for a PAR of 1:1:1:1 in the three receiving modes. The local reference signals used in the simulations for the three receiving modes are presented in Tab. 5, and the results have been multiplied by the speed of light for conversion into meters. It can be seen that the three modulations have almost the same code-tracking error. In the simulations, only the main lobes of the PSDs were included in the bandwidth, and for these three modulations, the PSDs have nearly identical main lobes. Thus, the results shown in Fig. 12 were derived. Note that for the FIC receiving mode, GTD-AltBOC and TD-AltBOC have higher multiplexing efficiencies than that of AltBOC, as shown in Tab. 8. As a result, with the use of a larger receiving bandwidth, GTD-AltBOC and TDAltBOC will achieve better code-tracking performance than AltBOC.

Moreover, Fig. 13 compares the code-tracking errors of GTD-AltBOC, ACE-BOC and GAltBOC for a PAR of 1:3:1:3 in the FIC and SBT receiving modes; the codetracking error of AltBOC is also plotted for comparison. Note that for the FMR receiving mode, all of the considered methods exhibit similar code-tracking performance and 


\begin{tabular}{|c|c|c|c|c|c|c|c|c|c|}
\hline \multirow{2}{*}{$\begin{array}{l}\text { Receiving } \\
\text { mode }\end{array}$} & \multicolumn{3}{|c|}{$1: 1: 1: 1$} & \multicolumn{3}{|c|}{$1: 3: 1: 3$} & \multicolumn{3}{|c|}{$1: 1: 3: 3$} \\
\hline & AltBOC & TD-AltBOC & GTD- & GTD- & ACE-BOC & GAltBOC & GTD- & ACE-BOC & GAltBOC \\
\hline FMR & 1 & 1 & 1 & 1 & 1 & 1 & 1 & 1 & 1 \\
\hline FIC & 0.8536 & 1 & 1 & 1 & 0.8720 & 0.75 & 1 & 0.8720 & 0.8796 \\
\hline SBT & 0.8106 & 0.8106 & 0.8106 & 0.8106 & 0.8106 & 0.6079 & 0.8106 & 0.8106 & 0.8353 \\
\hline
\end{tabular}

Tab. 8. Multiplexing efficiency for AltBOC, TD-AltBOC, GTD-AltBOC, ACE-BOC and GAltBOC (1:1:1:1, 1:3:1:3, 1:1:3:3)

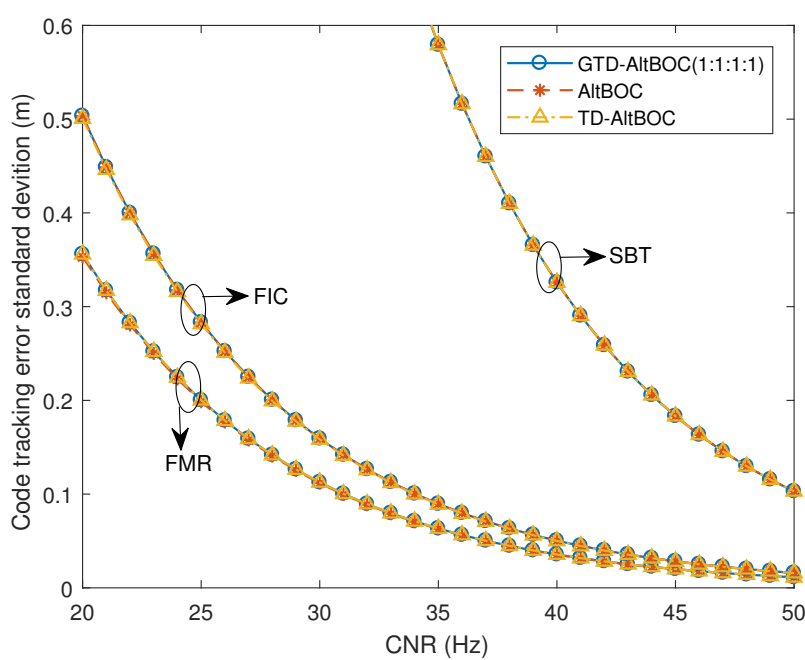

Fig. 12. Code-tracking errors of GTD-AltBOC, AltBOC and TD-AltBOC with a PAR of 1:1:1:1 for the three receiving modes.

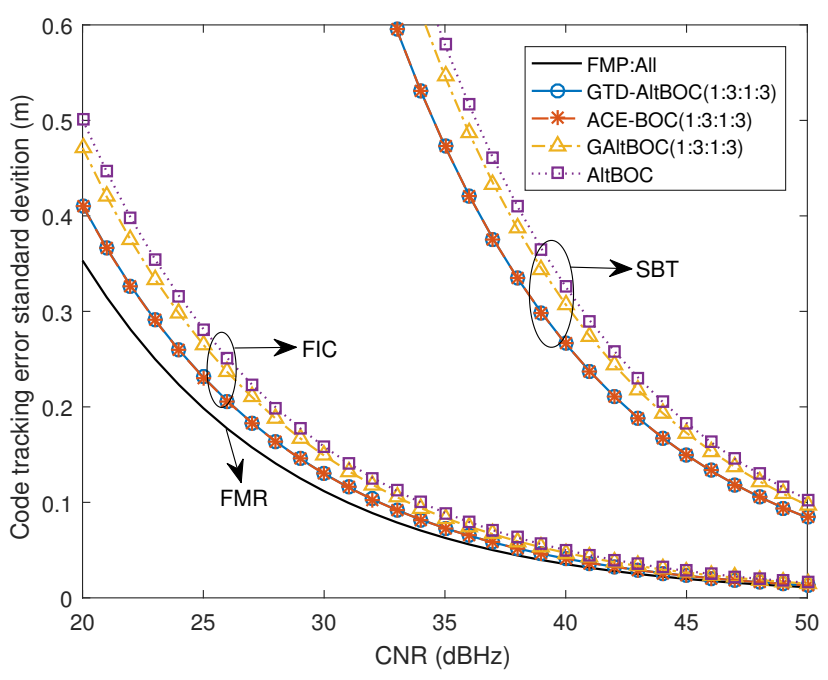

Fig. 13. Code-tracking errors of GTD-AltBOC, ACE-BOC and GAltBOC with a PAR of $1: 3: 1: 3$ and of AltBOC for the three receiving modes.

can all be described by one curve, shown as a black line in Fig. 13. Because of the higher pilot power, GTD-AltBOC, ACE-BOC and GAltBOC all show better code-tracking performance than AltBOC. GTD-AltBOC and ACE-BOC exhibit the best code-tracking performance in both the FIC and SBT receiving modes, whereas GAltBOC shows slightly worse code-tracking performance than that of GTD-AltBOC and ACE-BOC due to its lower multiplexing efficiency.

Figure 13 also illustrates the differences in codetracking performance among the different receiving modes.
The relatively wide receiving bandwidths for the FIC and FMP receiving modes result in a significant improvement in code-tracking performance compared with the SBT receiving mode, at the cost of an increased processing complexity. One advantage of the proposed GTD-AltBOC modulation method is that it allows the receiver processing complexity for the FIC and FMP receiving modes to be decreased in comparison with AltBOC, ACE-BOC and GAltBOC while maintaining high tracking performance. With further advancements in manufacturing capabilities, the FIC and FMR receiving modes will become promising choices for future receivers.

\section{Conclusions}

In this paper, we propose a general time-division dualfrequency constant envelope modulation technique called GTD-AltBOC. The principle of GTD-AltBOC modulation is investigated and three different time slot allocation schemes for the assignment of the signal components to even and odd time slots are discussed, namely, GTD-AltBOC-LU, GTD-AltBOC-DP and GTD-AltBOC-X. For the generation of GTD-AltBOC signals, we present a LUT-based signal generation architecture with high flexibility that facilitates hardware interoperability. For the reception of GTD-AltBOC signals, we present three typical receiving modes and analyze the complexity of each. We have also simulated the PSDs, correlation functions, multiplexing efficiencies and code-tracking errors of the proposed method for various PARs and receiving modes. Based on the principle, complexity analysis and the simulations, we summarize the following conclusions:

The proposed GTD-AltBOC method permit flexibility in the choice of the PAR. We can allocate more power to the pilot component to obtain a better code tracking performance or allocate more power to one sideband to provide better service in that sideband. This is a significant advantage of the proposed method over the original TD-AltBOC and TMOC-QPSK.

The storage and computational complexity of the proposed GTD-AltBOC modulation method are significantly reduced compared with those of AltBOC, ACE-BOC and GAlt$\mathrm{BOC}$ for the FIC and FMR receiving modes, whereas for the SBT receiving mode, GTD-AltBOC has a complexity similar to that of ACE-BOC and GAltBOC.

The simulated PSDs and correlation functions confirm that GTD-AltBOC enables successful combination of the four signal components at the designed frequency with the desired 
PAR. In the FIC mode, the multiplexing efficiency of the proposed method is higher than that of either ACE-BOC or GAltBOC; in the SBT mode, it is equal to that of ACE-BOC and higher than that of GAltBOC with a PAR of $1: \gamma^{2}: 1: \gamma^{2}$ but lower than that of GAltBOC with a PAR of $1: 1: \gamma^{2}: \gamma^{2}$. Simulations of the code-tracking error also demonstrate the high code-tracking performance of the proposed GTD-AltBOC modulation method.

Overall, the proposed GTD-AltBOC modulation method exhibits several significant advantages over existing methods. Not only can the PAR of the proposed method be adjusted to satisfy different requirements while achieving excellent performance, but the signals can also be easily generated and processed by satellite payload transmitters and receivers.

\section{Acknowledgments}

This work was supported by the National Natural Science Foundation of China (NSFC), Grant 61401171.

\section{References}

[1] EU: EUROPEAN GNSS (GALILEO). Open Service: Signal in Space Interface Control Document (OS SIS ICD). Issue 1.3, 2016, p. 4-7. [Online] Cited 2017-10-21. Available at: https://www.gsceuropa.eu/system/files/galileo_documents/Galileo-OS-SIS-ICD.pdf

[2] PELTON, N. J., MADRY, S., CAMACHO-LARA, S. Handbook of Satellite Applications. 2nd ed. Springer International Publishing, 2017. ISBN: 978-3-319-23386-4

[3] LESTARQUIT, L., ARTAUD, G., ISSLER, J.-L. AltBOC for dummies or everything you always wanted to know about AltBOC. In Proceedings of the 21st International Technical Meeting of the Satellite Division of The Institute of Navigation (ION GNSS 2008). 2008, p. 961-970. ISSN: 2331-5911

[4] TANG, Z., ZHOU, H., WEI, J., et al. TD-AltBOC: A new COMPASS B2 modulation. Science China-Physics Mechanics \& Astronomy, 2011, vol. 54, p. 1014-1021. DOI: 10.1007/s11433-011-4332-y

[5] ShIVARAMAIAH, N. C., DEMPSTER, A. G., RIZOS, C. TimeMultiplexed Offset-Carrier QPSK for GNSS. IEEE Transactions on Aerospace and Electronic Systems, 2013, vol. 49, no. 2, p. 1119-1138. DOI: 10.1109/TAES.2013.6494403

[6] WON, J. H., EISSFELLER, B., SCHMITZ-PEIFFER, A., et al. Trade-Off Between Data Rate and Signal Power Split in GNSS Signal Design. IEEE Transactions on Aerospace and Electronic Systems, 2012, vol. 48, no. 3, p. 2260-2281. DOI: 10.1109/TAES.2012.6237591

[7] FONTANA, R. D., CHEUNG, W., NOVAK, P. M., et al. The New L2 Civil Signal. In Proceedings of the 14th International Technical Meeting of the Satellite Division of The Institute of Navigation (ION GPS 2001). 2001, p. 617-631. ISSN: 2331-5911

[8] SPILKER, J. J., VAN DIERENDONCK, A. J. Proposed New Civil GPS Signal at $1176.45 \mathrm{MHz}$. In Proceedings of the 12th International Technical Meeting of the Satellite Division of The Institute of Navigation (ION GPS 1999). 1999, p. 1717-1726. ISSN: 2331-5911
[9] BETZ, J., BLANCO, M. A., CAHN, C. R., et al. Description of the L1C Signal. In Proceedings of the 19th International Technical Meeting of the Satellite Division of The Institute of Navigation (ION GNSS 2006). 2006, p. 2080-2091. ISSN: 2331-5911

[10] ZHANG, K. Generalised constant-envelope dualQPSK and altBOC modulations for modern GNSS signals. Electronics Letters, 2013, vol. 49, no. 21, p. 1335-1337. DOI: 10.1049/el.2013.2095

[11] YAO, Z., LU, M. Implementation, and Performance Analysis of ACEBOC Modulation. In Proceedings of the 26th International Technical Meeting of The Satellite Division of the Institute of Navigation (ION GNSS 2013). 2013, p. 361-368. ISSN: 2331-5911

[12] YAO, Z., ZHANG, J., LU, M. ACE-BOC: dual-frequency constant envelope multiplexing for satellite navigation. IEEE Transactions on Aerospace and Electronic Systems, 2016, vol. 52, no. 1, p. 466-485. DOI: 10.1109/TAES.2015.140607

[13] GUO, F., YAO, Z., LU, M. BS-ACEBOC: A generalized lowcomplexity dual-frequency constant-envelope multiplexing modulation for GNSS. GPS Solutions, 2017, vol. 21, no. 2, p. 561-575. DOI: $10.1007 / \mathrm{s} 10291-016-0547-8$

[14] YAN, T., WEI, J., TANG, Z., et al. General AltBOC Modulation with Adjustable Power Allocation Ratio for GNSS. The Journal of Navigation, 2016, vol. 69, no. 3, p. 531-560. DOI: $10.1017 / \mathrm{S} 037346331500079 \mathrm{X}$

[15] ShIVARAmAiAH, N. C., DEMPSTER, A. G., RIZOS, C. Hybrid tracking loop architectures for the Galileo E5 signal. In Proceedings of the European navigation conference on global navigation satellite systems (ENC GNSS 2009). 2009.

[16] DAFESH, P. A., CAHN, C. R. Phase-Optimized Constant-Envelope Transmission (POCET) Modulation Method for GNSS Signals. In Proceedings of the 22nd International Technical Meeting of The Satellite Division of the Institute of Navigation (ION GNSS 2009). 2009, p. 2860-2866. ISSN: 2331-5911

[17] BETZ, J. W., KOLODZIEJSKI, K. R. Generalized Theory of Code Tracking with an Early-Late Discriminator, Part I: Lower Bound and Coherent Processing. IEEE Transactions on Aerospace and Electronic Systems, 2009, vol. 45, no. 4, p. 1538-1550. DOI: 10.1109/TAES.2009.5310316

[18] TAWK, Y., BOTTERON, C., JOVANOVIC, A., et al. Analysis of Galileo E5 and E5ab code tracking. GPS Solutions, 2012, vol. 16, no. 2, p. 243-258. DOI: 10.1007/s10291-011-0226-8

\section{About the Authors...}

Zhihui ZHOU was born in Hunan, China, in 1988. He received his B.S. degree from Huazhong University of Science and Technology in 2011. He is currently working toward his Ph.D. degree in the School of Electronic Information and Communications, Huazhong University of Science and Technology, China. His research interests include next-generation GNSS signals and signal processing algorithms for GNSS receivers.

Zuping TANG (corresponding author) was born in Chongqing, China, in 1981. He received his B.S., M.Sc. and Ph.D. degrees from Huazhong University of Science and Technology, Wuhan, China, in 2002, 2005 and 2009, respectively. He is currently an associate professor with the School of Electronic Information and Communications, Huazhong University of Science and Technology. His research interests include GNSS signal design theory, signal quality evaluation and GNSS receiver techniques. 
Jiaolong WEI was born in Jiangxi, China, in 1965. He received his B.S., M.Sc. and Ph.D. degrees from Huazhong University of Science and Technology, Wuhan, China, in 1986, 1990 and 2004, respectively. He is currently a professor, Ph.D. supervisor and vice-dean of the School of Electronic Information and Communications, Huazhong University of Science and Technology. His research interests include wireless communications and networks, intelligent computation and satellite navigation.
Xuan XIA was born in Hubei, China, in 1987. He received his B.S. degree from Huazhong University of Science and Technology in 2009. He is currently working toward his Ph.D. degree in the School of Electronic Information and Communications, Huazhong University of Science and Technology, China. His research interests include next-generation GNSS signals and signal processing algorithms for GNSS receivers. 